\title{
Existence of Avian Infectious Bronchitis Virus with a European-Prevalent 4/91 Genotype in Japan
}

\author{
Masaji MASE ${ }^{1) *}$, Toshikazu INOUE ${ }^{1)}$, Shigeo YAMAGUCHI ${ }^{1)}$ and Tadao IMADA $^{1)}$ \\ 1) National Institute of Animal Health, 3-1-5 Kannondai, Tsukuba, Ibaraki 305-0856, Japan
}

(Received 4 June 2008/Accepted 7 July 2008)

ABSTRACT. Eight isolates of infectious bronchitis virus (IBV) were obtained from various prefectures in Japan during 2003-2007 and were genetically analyzed by reverse transcriptase-polymerase chain reaction (RT-PCR) coupled with direct sequencing. These IBV isolates were classified into three genetic groups, including two that have already been reported (JP-I and JP-III). The remaining group is related to the 4/91 (also known as 793/B) type, prevalent mainly in European countries, and has not been identified in Japan until now. KEY WORDS: epidemiology, genotype, infectious bronchitis virus.

Avian infectious bronchitis virus (IBV), a member of the Coronaviridae family (order Nidovirales, genus Coronavi$r u s$, is a highly contagious pathogen of domestic fowls worldwide. It replicates primarily in the respiratory tract but also in the epithelial cells of the gut, kidney and oviduct [4]. Coronaviruses are enveloped and positive-stranded RNA viruses containing an unsegmented genome approximately 27.6 kilobases $(\mathrm{kb})$ in length [1].

The major method of protecting poultry from this disease is the application of live or killed vaccines [4]. In the field, however, the protection afforded by vaccination is not complete, since the high mutation frequency of IBV leads to emergence of new viruses capable of causing disease in chickens [19]. IBV has three major virus-encoded structural proteins: the spike $(\mathrm{S})$ glycoprotein, membrane $(\mathrm{M})$ protein and nucleocapsid $(\mathrm{N})$ protein. The IBV spike is formed by post-translational cleavage of two separate polypeptide components, designated S1 and S2 [3]. Of these, the S1 glycoprotein is associated with virus attachment and is a major target of the neutralizing antibodies in chickens $[5,8]$, so serotypic evolution in IBV is associated primarily with the sequences of the $\mathrm{S} 1$ glycoprotein $[10,18]$. Therefore, recent genetic grouping of IBV has been performed mainly on the basis of the nucleotide sequences of the S1 glycoprotein gene $[9,11,12,17,18,20]$.

Previously, our phylogenetic analysis of mainly hypervariable (HVR) regions of S1 glycoprotein genes revealed that Japanese IBV strains could be classified into five genetic groups, one of which is indigenous to Japan and can not be placed within the existing groups in other countries [14].

In the present report, to define the epidemiology or relationships among recent IBV isolates in Japan during 20032007 , we used essentially the same procedure in an attempt to characterize eight Japanese IBV isolates from chickens.

\footnotetext{
* Correspondence to: Mase, M., Research Team for Zoonotic Diseases, National Institute of Animal Health, 3-1-5 Kannondai, Tsukuba, Ibaraki 305-0856, Japan.

e-mail:masema@affrc.go.jp
}

The IBV isolates were obtained from prefecture-based regional animal hygiene service centers in Japan (Table 1). Most of the IBV specimens were isolated by two or three passages using 10-day-old embryonated chicken eggs. The presence of IBV in the inoculated embryos was initially determined by IBV-specific reverse transcriptase-polymerase chain reaction (RT-PCR) as described previously [14] and by observation of characteristic embryonic changes such as dwarfing, stunting or curling. Also, a newly available commercial live attenuated IB vaccine strain $(\mathrm{GN})$, which was developed from an isolate from a chicken with nephritis in Japan, was used in this study. Viral RNA was extracted from infected allantoic fluids using an ISOGENLS kit (Nippon Gene, Tokyo, Japan). Reverse transcription, PCR amplification, sequencing and phylogenetic analysis were performed as described previously [14].

The expected sizes of DNA fragments were successfully amplified by RT-PCR from all the IBV samples in this study. Determination of the nucleotide sequences of the obtained PCR products revealed the diversity in their lengths (677-692 bp).

By phylogenetic analysis, the eight isolates were classified into three genetic groups; JP-I, JP-III and 4/91 (also known as 793/B; Fig. 1). Among them, two groups (JP-I and JP-III) had been identified in a previous study [14]. One isolate (JP/Ehime/2003), as well as the GN vaccine strain, both of which were classified into the JP-I genotype, were clustered only with the Japanese isolates, whereas four isolates (JP/Okayama-5/2004, JP/Chiba/2004, JP/Wakayama$13 / 2006$ and JP/Saitama/2007) classified into the JP-III genotype were clustered with isolates in neighboring countries such as South Korea and P.R.China. Three isolates (JP/ Wakayama/2003, JP/Okayama-7/2004 and JP/Saitama/ 2006) were related to genotype $4 / 91$ (793/B), which is prevalent mainly in European countries. In Japan, the 4/91-type live vaccine has been commercially available since 2002 , so we determined the nucleotide sequences of the corresponding regions of this vaccine strain and field isolates for comparison. The results showed that genetically JP/Okayama-7/ 
Table 1. IBV isolates from field cases in chickens during 2003-2007 in Japan

\begin{tabular}{lccccc}
\hline Virus & Isolation year & Type of chicken & Major clinical sign & Tissue & Genetic Group \\
\hline JP/Ehime/2003 & 2003 & Broiler breeder & Depression, respiratory & Trachea & JP-I \\
JP/Wakayama/2003 & 2003 & Broiler & Depression, diarrhea & Trachea & $4 / 91$ \\
JP/Okayama-5/2004 & 2004 & Layer & Egg drop & Rectum & JP-III \\
JP/Okayama-7/2004 & 2004 & Broiler breeder & Nephritis & Kidney & $4 / 91$ \\
JP/Chiba/2004 & 2004 & Broiler & Nephritis & Kidney & JP-III \\
JP/Saitama/2006 & 2006 & Layer & Respiratory & Trachea plus rectum & $4 / 91$ \\
JP/Wakayama-13/2006 & 2006 & Broiler & Rise in mortality & Feces & JP-III \\
JP/Saitama/2007 & 2007 & Layer & Egg drop & rectum & JP-III \\
\hline
\end{tabular}

2004 was highly similar to the vaccine strain, with a similarity of over $99 \%$, and so this strain may have been derived from the vaccine strain. However, the remaining two isolates (JP/Wakayama/2003 and JP/Saitama/2006) were most similar to a French isolate (FR-94047-94, Accession Number AJ618987), with a similarity of $96.8 \%$ by GenBank searches, and they had similarities of 93.3 and $93.8 \%$, respectively, compared with the vaccine strain; these isolates were not likely to be directly derived from the introduced vaccine strain.

In a previous study, we reported the simple and rapid classification of genotypes of Japanese IBV strains using two restriction endonucleases, Hae II and Eco RI. The strains belonging to the JP-I and JP-III genotypes showed the same profiles as reported previously. However, the newly identified genotype 4/91 (793/B) did not have sites for these 2 enzymes like the Mass type, so differentiation between the 4/91 and Mass types was difficult. Therefore, we selected another endonuclease, Pst I, to differentiate between the 4/91 and Mass types. The strains belonging to the 4/91 type do not have a restriction site for this enzyme, but the strains belonging to the Mass type do. For example, the PCR product of $\mathrm{H} 120$ origin, which is a representative Mass-type strain, was digested with 583 and 88 base pairs (data not shown). These results revealed that differentiation of Japanese IBV strains is possible at present by use of three restriction endonucleases.

The present study shows that the 4/91 (793/B) type of IBV has existed in Japan since 2003. Since it was first identified in France in 1985 and subsequently identified in Great Britain in 1990, this genotype has spread to many countries and become one of the most predominant types in Europe $[2,6,7]$. This IBV genotype has also been detected in Middle Eastern and Asian countries, such as Iran and Thailand $[6,16]$. However, this genotype was not found in our previous report using isolates from up to mid-2003 [14], in which we reported that the recent IBV isolates in Japan could be classified into three major genotypes (JP-I, JP-II and JP-III).

The introduction route of the 4/91 genotype into Japan is unknown. However, highly virulent infectious bursal disease virus or avian pneumovirus are known to be examples of avian viruses detected in Japan that are genetically similar to those prevalent in Europe $[13,15]$. The importation of chicks from European countries, such as the UK, France, the Netherlands and Germany is extensive in Japan; over
500,000 chicks were imported to Japan each year between 2002 and 2006. This may be one of the transfer routes for the 4/91 genotype, and other IBV genotypes may be introduced to Japan in the same way. Detection of the novel 4/91 genotype in this study suggests that continuous isolation and genetic analysis of IBV is required. Furthermore, to understand the epidemiology of IBV in Japan, it will be important to know the prevalence of viruses not only in neighboring countries but also around the world.

ACKNOWLEDGMENTS. We would like to thank the veterinary officials of Ehime, Wakayama, Okayama, Chiba and Saitama Prefectures for their cooperation in collection of the viral samples.

\section{REFERENCES}

1. Boursnell, M. E., Brown, T. D., Foulds, I. J., Green, P. F., Tomley, F. M. and Binns, M. M. 1987.Completion of the sequence of the genome of the coronavirus avian infectious bronchitis virus. J. Gen. Virol. 68: 57-77.

2. Capua, I., Minta, Z., Karpinska, E., Mawditt, K., Britton, P., Cavanagh, D. and Gough, E. 1999.Co-circulation of four types of infectious bronchitis(793/B, 624/I, B1648 and Massachusetts). Avian Pathol. 28: 587-592.

3. Cavanagh, D. 1983.Coronavirus IBV: further evidence that the surface projections are associated with two glycopolypeptides. J. Gen. Virol. 64: 1787-1791.

4. Cavanagh, D. and Naqi, S. 2001. pp. 101-119. Infectious Bronchitis. Diseases of Poultry, 11 th edn. Ames, IA: Iowa State University Press.

5. Cavanagh, D., Davis, P. J. and Mockett, A. P. 1988.Amino acids within hypervariable region 1 of avian coronavirus IBV (Massachusetts serotype) spike glycoprotein are associated with neutralization epitopes. Virus Res. 11: 141-150.

6. Cook, J. K., Orbell, S. J., Woods, M. A. and Huggins, M. B. 1996.A survey of the presence of a new infectious bronchitis virus designated 4/91 (793B). Vet. Rec. 138: 178-180.

7. Dolz, R., Pujols, J., Ordonez, G., Porta, R. and Majo, N. 2008. Molecular epidemiology and evolution of avian infectious bronchitis virus in Spain over a fourteen-year period. Virology 374: 50-59.

8. Kant, A., Koch, G., van Roozelaar, D. J., Kusters, J. G., Poelwijk, F. A. and van der Zeijst, B. A. 1992.Location of antigenic sites defined by neutralizing monoclonal antibodies on the S1 avian infectious bronchitis virus glycopolypeptide. J. Gen. Virol. 73: 591-596.

9. Keeler, C. L., Jr., Reed, K. L., Nix, W. A. and Gelb, J., Jr. 


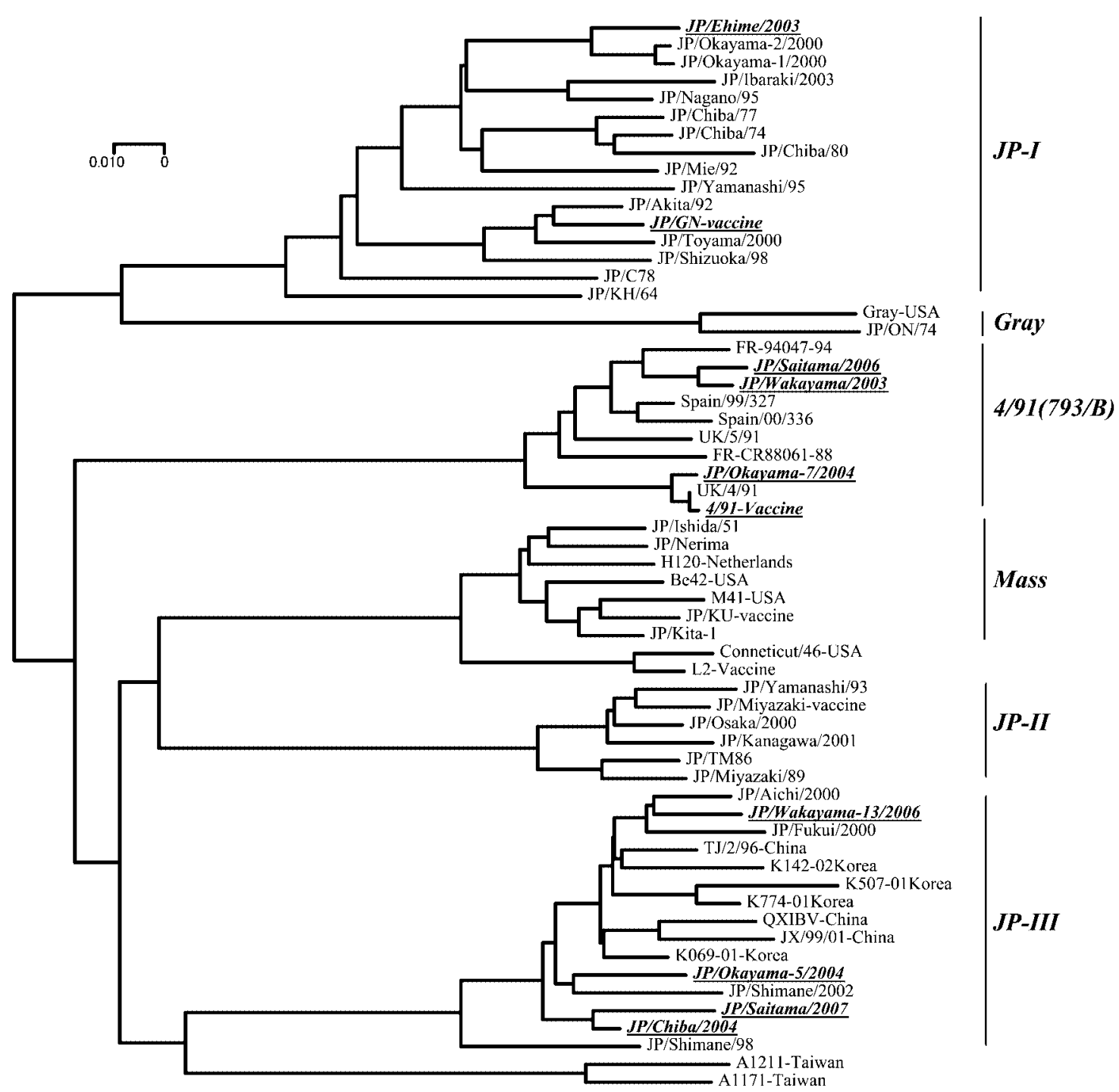

Fig. 1. Phylogenetic tree based on the S1 glycoprotein gene of IBV. Nucleotides 20368-20988 (621 bases) of the S1 gene of IBV Beaudette (GenBank Accession No. NC001451) were subjected to phylogenetic analysis. Horizontal distances are proportional to the minimum number of nucleotide differences required to join nodes and sequences. The viruses employed in this study are italicized and underlined.

1998. Serotype identification of avian infectious bronchitis virus by RT-PCR of the peplomer (S-1) gene. Avian Dis. 42: 275-284.

10. Kusters, J. G., Niesters, H. G., Lenstra, J. A., Horzinek, M. C. and van der Zeijst, B. A. 1989.Phylogeny of antigenic variants of avian coronavirus IBV. Virology 169: 217-221.

11. Lee, C. W., Hilt, D. A. and Jackwood, M. W. 2003.Typing of field isolates of infectious bronchitis virus based on the sequence of the hypervariable region in the $\mathrm{S} 1$ gene. J. Vet. Diagn. Invest. 15: 344-348.

12. Lee, S. K., Sung, H. W. and Kwon, H. M. 2004.S1 glycoprotein gene analysis of infectious bronchitis viruses isolated in Korea. Arch. Virol. 149: 481-494.

13. Lin, Z., Kato, A., Otaki, Y., Nakamura, T., Sasmaz, E. and Ueda, S. 1993. Sequence comparisons of a highly virulent infectious bursal disease virus prevalent in Japan. Avian Dis. 37: 315-323.

14. Mase, M., Tsukamoto, K., Imai, K. and Yamaguchi, S. 2004.Phylogenetic analysis of avian infectious bronchitis virus strains isolated in Japan. Arch. Virol. 149: 2069-2078.

15. Mase, M., Yamaguchi, S., Tsukamoto, K., Imada, T., Imai, K. and Nakamura, K. 2003.Presence of avian pneumovirus subtypes A and B in Japan. Avian Dis. 47: 481-484.

16. Seyfi Abad Shapouri, M. R., Mayahi, M., Assasi, K. and Charkhkar, S. 2004.A survey of the prevalence of infectious bronchitis virus type 4/91 in Iran. Acta. Vet. Hung. 52: 163166.

17. Wang, C. H. and Tsai, C. T. 1996.Genetic grouping for the isolates of avian infectious bronchitis virus in Taiwan. Arch. Virol. 141: 1677-1688. 
18. Wang, C. H. and Huang, Y. C. 2000.Relationship between serotypes and genotypes based on the hypervariable region of the $\mathrm{S} 1$ gene of infectious bronchitis virus. Arch. Virol. 145: 291-300.

19. Wang, L., Junker, D. and Collisson, E. W. 1993.Evidence of natural recombination within the S1 gene of infectious bronchi- tis virus. Virology 192: 710-716.

20. Yu, L., Wang, Z., Jiang, Y., Low, S. and Kwang, J. 2001.Molecular epidemiology of infectious bronchitis virus isolates from China and Southeast Asia. Avian Dis. 45: 201209. 